\title{
BMJ Open Study protocol for a mixed methods prospective cohort study to explore experiences of care following a suicidal crisis in the Australian healthcare system
}

\author{
Hannah Rosebrock (D) , ${ }^{1}$ Nicola Chen, ${ }^{1}$ Michelle Tye, ${ }^{1,2}$ Andrew Mackinnon, ${ }^{1}$ \\ Alison L Calear, ${ }^{3}$ Philip J Batterham, ${ }^{3}$ Myfanwy Maple, ${ }^{4}$ Victoria-Mae Rasmussen, ${ }^{1}$ \\ Liz Schroeder, ${ }^{5}$ Henry Cutler, ${ }^{5}$ Fiona Shand ${ }^{1,2}$
}

To cite: Rosebrock H, Chen N, Tye M, et al. Study protocol for a mixed methods prospective cohort study to explore experiences of care following a suicidal crisis in the Australian healthcare system. BMJ Open 2020;10:e033814. doi:10.1136/ bmjopen-2019-033814

- Prepublication history for this paper is available online. To view these files, please visit the journal online (http://dx.doi. org/10.1136/bmjopen-2019033814).

HR and NC are joint first authors

Received 27 August 2019

Revised 13 May 2020

Accepted 29 May 2020

Check for updates

(C) Author(s) (or their employer(s)) 2020. Re-use permitted under CC BY-NC. No commercial re-use. See rights and permissions. Published by BMJ.

For numbered affiliations see end of article.

Correspondence to Hannah Rosebrock; h.rosebrock@blackdog.org. au and

Dr Fiona Shand;

Fionas@unsw.edu.au

\section{ABSTRACT}

Introduction For individuals presenting to the emergency department (ED) for a suicide attempt, the period after discharge from hospital is marked by heightened vulnerability for further suicide attempts. Effective care following a suicidal crisis has the potential to significantly decrease this risk. The current study aims to examine the impact of the LifeSpan multilevel suicide prevention model on experiences of care following a suicidal crisis. Perspectives from healthcare consumers (individuals who have presented to the ED following a suicidal crisis), carers, and health professionals will be explored. The LifeSpan model is currently being evaluated as a highfidelity trial in four geographically defined regions in New South Wales, Australia.

Methods and analysis This study will use a mixed methods prospective cohort design. Quantitative data collection includes a structured survey, administered to healthcare consumers from LifeSpan sites and control sites. Two cohorts of healthcare consumers will be recruited 12 months apart with baseline assessment occurring within 18 months of the ED presentation, and follow-up 12 months after the initial assessment. Survey participants will be recruited online and through participating EDs, mental health organisations and aftercare services. Qualitative interview data from healthcare consumers, carers who have accompanied a loved one to the ED following a suicidal crisis and health professionals who provide care to people at risk of suicide will be collected concurrently with the recruitment of the first cohort of survey participants. Purposive and convenience sampling techniques will be used for recruitment of interview participants. The primary outcome for this study will be healthcare consumers' experiences of service provided at the ED. Analysis will be undertaken of the change over time within LifeSpan sites, as well as between LifeSpan sites and control sites, using mixed effects repeated measures models as principal means of data analysis.

Ethics and dissemination This research has been approved by the Hunter New England Human Research Ethics Committee (HREC/17/HNE/144). Results will
Strengths and limitations of this study

- Prospective cohort design in which data are collected from LifeSpan sites and control sites which will allow to determine the impact of LifeSpan on healthcare experiences of consumers.

- This is a mixed methods study which includes a qualitative component to explore in depth the elements of effective care following a suicidal crisis, as well as gaps, issues and areas for improvement.

- This study follows global trends and national policy directions calling attention to the inclusion of persons with a lived experience in service design, planning and delivery.

- Given the national emphasis on improving care for people at risk of suicide some control sites have started undertaking service improvement measures in line with the LifeSpan Crisis Care and Aftercare Strategy (ie, establishment of an aftercare service in one of the control sites).

be disseminated via conferences and peer-reviewed journals.

Trial registration number ACTRN12617000457347.

\section{INTRODUCTION}

Suicide is a significant and increasing problem in Australia, with rates of suicide in 2017 being the highest in the past decade, equal with 2015. ${ }^{1}$ In 2017, a total of 3128 people died by suicide. For every suicide death, there are 20-30 suicide attempts. ${ }^{23}$

A previous suicide attempt has been identified as one of the strongest risk factors for subsequent suicide in Australia ${ }^{4}$ and internationally. ${ }^{5-7}$ The risk is greatest immediately after discharge from an emergency department (ED)/psychiatric ward and can remain high for more than 12 months after the 


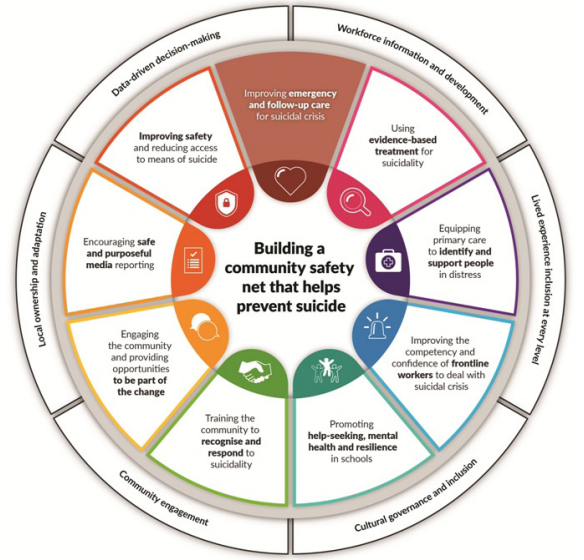

Figure 1 LifeSpan suicide prevention model.

attempt. ${ }^{89}$ In a recent meta-analysis the authors reported that the risk of suicide for individuals discharged from psychiatric facilities after a suicide-related admission was more than four times the risk of other samples (2078 vs 452 per 100000 person-years). ${ }^{10}$ It has been estimated that effective care after a suicide attempt has the potential to decrease suicide attempts by $19.8 \%$ and suicide deaths by $1.1 \% .^{11}$ Hence, the provision of high-quality care at the ED, and continuity of care after discharge forms an essential component of suicide prevention. Despite the urgent need to develop evidence-based prevention, few studies have explored patients' experiences of care following a suicidal crisis (including attempt, thoughts and/or ideation of suicide). Only limited support was found for patient satisfaction with care. In the Australian context, frequently reported issues experienced by patients included difficulties in accessing services, poor staff attitudes, not being involved in treatment decisions and being discharged too early. ${ }^{12} 13$ Furthermore, patients reported not having their emotional distress attended to and being left to seek their own options for help after discharge. ${ }^{12}$ Satisfaction with emergency care appears to be particularly low. This is alarming given that the ED is the first point of contact for many individuals who experience a suicidal crisis. $^{12}$

The LifeSpan Integrated Suicide Prevention Trial is the first multilevel approach to suicide prevention to be tested in Australia (figure 1). Multilevel models of suicide prevention emerged from Europe, where the Nuremberg Alliance Against Depression and the European Alliance Against Depression produced significant reductions in suicidal behaviour. ${ }^{14} 15$ The LifeSpan model was developed from a scoping review of evidence-based suicide prevention strategies in 2015. LifeSpan incorporates nine evidence-based strategies, delivered within the same 2-year time frame to community, schools and healthcare systems. Four geographically defined regions in New South Wales (NSW), Australia, which have a high incidence of suicide are implementing LifeSpan as part of a randomised stepped-wedge high-fidelity trial. Local coordination and implementation are supported by a regional collaboration, and by a team at the Black Dog Institute, Sydney, Australia. The LifeSpan strategies are tailored to each site which considers site-specific features to assist implementation. Primary outcomes from the LifeSpan trial will not be available until 2021. ${ }^{16}$

The LifeSpan Crisis Care and Aftercare Strategy aims to review and improve care following a suicidal crisis. Crisis care is defined as the care after the immediate medical response and stabilisation, but before the provision of ongoing therapeutic treatment. ${ }^{17}$ In this study it is operationalised as the care provided at the ED. Aftercare is defined as the coordinated follow-up care on agreed treatment plans, which aims to increase access to and engagement in care to prevent suicide and related behaviours. ${ }^{1718}$ To support and coordinate improvement of care sites will be provided with a set of evidence-based lived experience informed guidelines developed through a rigorous Delphi consensus study. ${ }^{19} 20$ Importantly, these guidelines recommend for all individuals who have presented to the ED following a suicidal crisis to be offered the opportunity to have a carer involved in assessment and treatment, to receive a comprehensive psychosocial assessment and to be followed up after discharge. Further, LifeSpan sites will be supported with the establishment of an aftercare service.

This study aims to examine the impact of LifeSpan on experiences of care following a suicidal crisis. Perspectives from healthcare consumers (individuals who have presented to the ED following a suicidal crisis), carers (support persons or family members who have accompanied a loved one to the ED for a suicide-related presentation) and health professionals (ED clinicians, mental health nursing staff, community mental health clinicians and aftercare support workers who provide professional support or treatment to persons at risk of suicide) will be explored. Primary and secondary aims for this study, as well as hypotheses are presented in table 1 .

\section{METHODS}

\section{Study design}

The LifeSpan model is delivered to sites in the context of a stepped-wedge cluster randomised trial design. Sites are being assigned at random to crossover from their current practices to the 2-year active implementation period of LifeSpan. Crossovers occur in intervals of 4 months between sites and are followed by a 6-month establishment period. The intent is to have interventions in line with the nine LifeSpan strategies all implemented in each site by the end of its 2-year active implementation period. Each site determines the order in which each of the nine strategies is implemented, dependent on local needs and capacity.

The current study is nested within the broader LifeSpan Suicide Prevention Trial and will use a mixed methods prospective cohort design in which data will be collected from LifeSpan trial sites and control sites. The prospective cohort design will allow the researchers to 
Table 1 Study aims and hypotheses

\begin{tabular}{|c|c|c|}
\hline Aim & Description & Hypothesis \\
\hline & & $\begin{array}{l}\text { At } 12 \text { and } 24 \text { months after baseline consumers who } \\
\text { have been exposed to LifeSpan, but not consumers } \\
\text { who have not been exposed, will report: }\end{array}$ \\
\hline Primary (i) & $\begin{array}{l}\text { To compare change over time in the healthcare } \\
\text { experiences of consumers presenting to EDs in } \\
\text { LifeSpan trial sites following a suicidal crisis to the } \\
\text { experiences of consumers presenting to EDs in control } \\
\text { sites. }\end{array}$ & $\begin{array}{l}\text { More positive healthcare experiences, as measured } \\
\text { by the Your Experience with Service (YES) survey. }\end{array}$ \\
\hline Secondary (ii) & $\begin{array}{l}\text { To examine healthcare consumer self-report measures } \\
\text { of (1) comprehensiveness and content of the } \\
\text { assessment undertaken at the ED, (2) opportunities of } \\
\text { carer involvement in assessment and treatment, and } \\
\text { (3) discharge and follow-up processes. }\end{array}$ & $\begin{array}{l}\text { 1. Greater comprehensiveness of the assessment } \\
\text { undertaken at the ED. } \\
\text { 2. More opportunities for carer involvement in } \\
\text { assessment and treatment. } \\
\text { 3. Increased rate of follow-up after discharge from } \\
\text { the ED. }\end{array}$ \\
\hline Secondary (iv) & $\begin{array}{l}\text { To explore the ED response to suicidal crisis in depth } \\
\text { through qualitative inquiry into healthcare consumers, } \\
\text { carers and health professionals' perspectives of care } \\
\text { provided following a suicidal crisis. }\end{array}$ & N.A. \\
\hline
\end{tabular}

ED, emergency department; N.A., not applicable.

track participants' ED experiences over time with analysis being undertaken of the change in experiences during the roll-out of LifeSpan between trial sites and control sites. As a randomised controlled trial was not feasible for the current study (see the Discussion section), the prospective cohort design was selected, as it provides the next strongest level of evidence. ${ }^{21}$ Importantly, the inherent temporal dimension will enable the researchers to examine the direction of effects. ${ }^{22}$ The qualitative component of this study will allow to explore the more complex aspects of the healthcare system in depth-such as patient experiences of care-and as such contribute crucially and in a unique way to the quantitative data collection. ${ }^{23} 24$ The mixed methods design will result in greater completeness of the data with each method enhancing understanding generated with the other. ${ }^{23}$

\section{Quantitative data collection}

Data to address aims (i), (ii) and (iii) will be collected through an anonymous structured survey from individuals who have presented to the ED following a suicidal crisis. Given low rates of re-presentation to the $\mathrm{ED}^{36}$ two cohorts will be recruited, 12 months apart to collect additional data. The first cohort will be recruited during the 2-year active implementation period of LifeSpan and will include ED presentations from the previous 18 months. The second cohort will include ED presentations up to 12 months prior. Follow-up for both cohorts will occur 12 months after initial recruitment meaning that follow-up of cohort 1 will occur simultaneously with recruitment of cohort 2 (figure 2).

\section{Qualitative data collection}

To address aim (iv), semistructured qualitative interviews will be conducted with three groups of participants: (1) healthcare consumers; (2) carers; and (3) health professionals. Where possible participants will be followed up 12 months after the initial interview. Interview data will not be paired with survey data.

\section{Study setting}

The sites for the implementation of the LifeSpan model were selected based on an expression of interest and an identified need for increased suicide prevention, matched against set criteria (ie, demographics, population size, number and size of public hospitals with an ED). ${ }^{25}$ Following selection of each LifeSpan site, a control site was chosen that falls within the boundaries of a single local health district (LHD). In Australia, LHDs are responsible for the management of public hospitals and health institutions and for providing public health

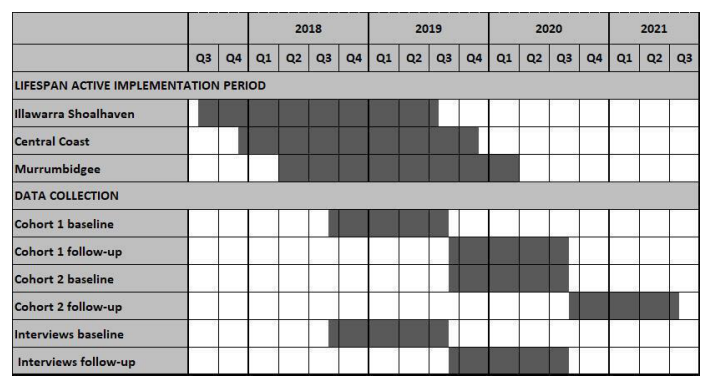

Figure 2 Study design overview: recruitment periods and LifeSpan active implementation phases. 
Table 2 Site characteristics

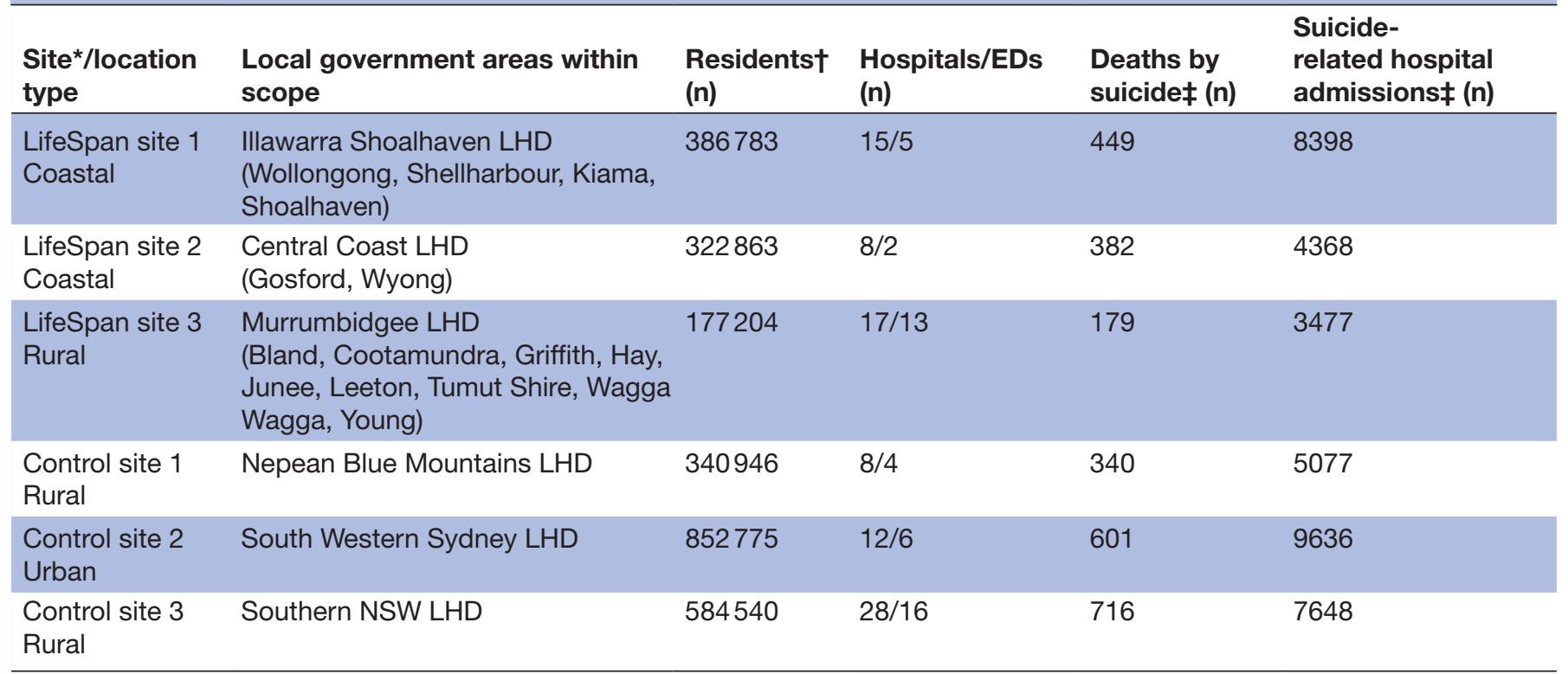

*The fourth LifeSpan site, Newcastle LHD, has not been included in the current study, due to separate studies targeting the same participant groups are being conducted in the same time frame. This is to not overburden an already vulnerable group of participants.

†Rate per 100.000 .

$\ddagger 2007-2016$ (10 years).

ED, emergency department; LHD, local health district.

services within a geographically defined area of the state. Relevant characteristics of LifeSpan sites and control sites are summarised in table 2.

\section{Exposure condition: the LifeSpan Crisis Care and Aftercare Strategy}

This strategy involves multiple technical, behavioural and organisational components. Sites will lead the implementation process, with the Black Dog Institute providing support. The precise operationalisation of the strategy will be tailored to site-specific needs and will be dependent on local differences in resource availability, governance structures and stakeholder engagement. To coordinate the process of reviewing and improving care provided following a suicidal crisis, all LifeSpan sites will be provided with the 'Guidelines for integrated suiciderelated crisis and follow-up care in Emergency Departments and other acute settings', ${ }^{20}$ hence referred to as the Guidelines. The Guidelines were developed using the Delphi consensus method which involved experts from various health disciplines and individuals with lived experience of suicide. ${ }^{19}$ They represent a detailed consolidation of the most essential recommendations contained in published original research, systematic reviews and guidelines for crisis care. Importantly, the Guidelines recommend for all individuals presenting to the ED following a suicidal crisis to receive a comprehensive psychosocial assessment, rather than exclusively providing this assessment to those considered to be 'high-risk'. The comprehensive psychosocial assessment places emphasis on building rapport with the patient and includes clear communication to the patient about the function of the assessment. The Guidelines further recommend involvement of the individuals' carers, support persons or family members in the care process with consent of the patient, and to offer assertive follow-up care after discharge. 'Assertive' in this context means that the responsibility for getting in touch and maintaining contact after discharge lies with the health professional (ie, the person's case manager) rather than with the individual at risk.

For all sites, the operationalisation of the Crisis Care and Aftercare will include local LHDs conducting internal audits of their services against the Guidelines, followed by a gap analysis to identify areas for improvement and the identification of barriers and facilitating factors addressing the identified gaps. Local working groups will be established with the ability to drive the implementation of specific components of the Guidelines selected during the gap analysis. Sites will decide which components of the Guidelines to implement, and as such implementation activities might differ slightly from site to site. Crucially, establishment of an aftercare service will be facilitated within each LifeSpan site, which will include assertive outreach, solution-focused counselling, support to adhere to treatment and, where possible, continuity of contact with the same staff member for up to 12 weeks. Progressive implementation of strategies to review and improve existing care will occur throughout the 2 years and will be managed locally by trial sites with the LifeSpan research team providing support. 


\section{Participants and recruitment}

\section{Survey participants}

Healthcare consumers will be eligible to participate in the structured anonymous survey if they are aged 16 and over, are located in Australia within one of the LifeSpan sites or control sites and have presented to an ED located within one of the LifeSpan sites or control following a suicidal crisis within 18 months prior to recruitment (cohort 1) or within 12 months prior to recruitment (cohort 2).

Healthcare consumers will be recruited online, via Facebook advertisements, and through participating EDs, mental health organisations and aftercare services. Facebook allows for the targeting of participants based on age range and geographical region. In this study the advertisements will be displayed to any person residing within a LifeSpan region or a control region, aged 16 years and over. By clicking the advertisement individuals will be redirected to an anonymous online survey. Individuals will be asked to provide informed consent before commencing the surveys. At the end of the survey participants will have the option to leave their contact details for follow-up research. Advertisements will be live on Facebook for approximately 1 month during recruitment periods.

Face-to-face recruitment will include the dissemination of study information flyers and/or paper copies of the structured anonymous survey in return envelopes including the participant information and consent forms. Recruitment will target major tertiary hospitals, smaller regional hospitals, and community-based services and organisations, including EDs, mental health inpatient units, community health, general/specialist medical practices and allied health practices/organisations, and aftercare services within each site for which research governance approval has been obtained. This includes all three LifeSpan sites included in this study but only one of the control sites. The study information flyer contains general information about the study and contact details for the research team, and states eligibility criteria and options for participation. Individuals can choose to (1) access the online version of the survey via the survey link or QR code included in the flyer; or (2) request a paper copy through contacting the research team.

Dissemination strategies will include the display of the study information flyer in waiting areas, inclusion in discharge packs and direct distribution of the flyer and/ or the paper version of the survey by healthcare staff to eligible participants. Healthcare staff will not administer surveys, all responses will be self-completed.

\section{Interview participants}

Three groups of participants will be recruited to participate in a semistructured interview:

1. Healthcare consumers: A subset of survey participants who have provided their contact details for further research will be invited to participate in an interview via email. Purposive sampling techniques will be used to ensure representation of individuals who attempted suicide and individuals who experienced suicidal thoughts but did not attempt suicide, as well as representation of a variety of hospitals.

2. Carers: Carers will be eligible to participate in a qualitative interview if they are aged 16 and over, are located in Australia within one of the trial sites or control sites and have accompanied a loved one to the ED following a suicidal crisis in the past 18 months. Carer invitations will be included in invitations to healthcare consumers. Additionally, participant information and consent forms and study information flyers will be sent to suicide lived experience groups/networks within approved sites inviting carer participation. Based on information provided in the form and flyer, carers can register their interest to participate in an interview by contacting the research team via email/phone/mail.

3. Health professionals: Health professionals will be eligible to participate in a qualitative interview if they are aged 16 and over, are located in Australia within one of the trial sites or control sites and are involved with the care for individuals at risk of suicide. Convenience sampling techniques will be used for recruitment of health professionals for interviews. Site contacts in both LifeSpan sites and control sites will be asked to point out the study to eligible health professionals and to hand out information and consent forms.

\section{Patient and public involvement}

A lived experience advisory committee has been established consisting of three individuals with a lived experience of suicide to provide ongoing advice to the study. This includes advice on design of recruitment materials, determining questions for the qualitative interviews and dissemination of results. Furthermore, people with a lived experience of suicide contributed significantly to the development of the Guidelines ${ }^{19} 20$ which form an integral part of the LifeSpan Crisis Care and Aftercare Strategy.

\section{Data collection and management}

\section{Quantitative outcomes}

The structured quantitative survey will be administered predominantly as an online questionnaire. Participants will have access to the secure online survey via a sitespecific link disseminated via study recruitment materials and Facebook advertisements. Data will be extracted and loaded into the LifeSpan Black Dog Institute data environment, hosted on a secure server maintained by the University of New South Wales. Any surveys completed with pen and paper will be entered manually into the LifeSpan Black Dog Institute data environment and destroyed once digitalised.

\section{Interview data}

Interviews will be conducted face to face at a location of the participants' choice, via video call using secure conferencing software or via telephone, depending on feasibility and participant preferences. Interviewers will have a counselling or psychology background. Interviews 
will be audio recorded with permission of the participant, transcribed and deidentified (names and other identifying information will be omitted). Audio records of qualitative interviews will be stored as password-protected electronic files. Transcripts of interviews will be deidentified and stored in the Black Dog Institute data environment. In direct quotes participants will be identified by age and gender only. Participants will be granted access to their transcribed data on request.

\section{Measures}

\section{Quantitative outcomes}

The measures listed below will be included in the survey and will be taken at each data collection period (ie, at baseline and at 12 months of follow-up) with the exception of omitting most demographic questions at 12 months of follow-up. In addition to the scales listed below, the survey also includes items to be used in the economic modelling for the overall LifeSpan intervention (Refs: 16/09/21/4.05). Psychometric properties of included measures are provided where available. At 12 months of follow-up participants who have not revisited the ED for a suicide-related presentation in the past 12 months will be able to skip the ED-related items and scales listed under 'Primary outcome' and 'Secondary outcomes'.

Demographic factors: age, gender, sexual orientation, current relationship status, ethnicity, language spoken at home, education, employment, history of psychiatric diagnoses.

\section{Primary outcome}

Your Experience of Service survey (YES, modified, shortened): A subset of seven partly modified questions of the YES survey will be used to capture information on consumers' experience of the ED for a suicide-related presentation. The YES in its original form assesses mental health consumers' experience of care and is endorsed by the Australian Government Department of Health. ${ }^{26}$ Items (eg, 'You felt welcome at this service') are scored on a 4-point Likert scale ranging from 'Strongly Disagree' to 'Strongly Agree'.

\section{Secondary outcomes}

Data on other service characteristics will also be collected (eg, length of stay, waiting times), as well as data related to the quality of the psychosocial assessment, handover processes and the discharge process.

Assessment at the ED: A 15-item scale has been constructed by the authors to assess the comprehensiveness of the assessment conducted at the ED with individuals presenting following a suicidal crisis. The aim of this scale is to provide an estimate of the extent to which a comprehensive psychosocial assessment has been performed at the ED, as recommended by the Guidelines. ${ }^{20}$ Participants are presented with a list of items, for example, 'Reason for presentation at ED', 'Home life', 'Friendships', and are asked to rate how much information ED staff asked them about those items on a 4-point Likert scale, ranging from 'None' to 'Lots/Substantial'.

Participants are also asked whether they were given enough time to talk and whether a family member, carer or friend was asked to be involved in the assessment.

Follow-up care: Data will be collected regarding the arrangement of follow-up appointments (if any) in the $\mathrm{ED}$, the nature of those appointments, whether the participant attended the appointment(s), the time frame between discharge and follow-up appointment, whether the participant felt there was sufficient information provided to the person who conducted the follow-up appointment as related to the participant and the perceived helpfulness of those appointments.

Aftercare: Data will be collected regarding whether the participant received a referral to an aftercare service, the nature of this aftercare service, whether the participant accepted the aftercare referral, the person who contacted the participant from the aftercare service, the helpfulness of the aftercare programme, whether the participant would recommend the aftercare programme to other people in suicidal crisis and suggestions for improvement.

\section{Other outcomes}

Columbia Suicide Self-Report Scale (C-SSRS—self-report, modified): A modified version of the Columbia Suicide Severity Rating Scale-self-report screener will be used to determine the presence or absence of suicidal ideation and behaviours, as well as the severity of suicidal ideation in the past 6 months. Participants are asked about the frequency and duration of suicidal thoughts and the extent to which they can control them, the intention to act on these thoughts and recent suicide attempts. It includes branching logic which requires participants to complete between 5 and 10 questions, depending on their responses. The original C-SSRS self-report instrument has been shown to predict risk of subsequent suicidal behaviour. ${ }^{27} 28$

Actual Help-Seeking Scale: A modified version of the Actual Help-Seeking Questionnaire will be used to assess people's help-seeking experiences in the last 6 months. ${ }^{29}$ Participants are presented a list of formal and informal help sources and asked from which source they have sought help for a personal/emotional/mental health problem, and to rate how helpful they found that source of help on a 5-point Likert scale from 'not helpful at all' to 'extremely helpful'. Additional sources of help can be added by the participant if needed.

Stigma of Suicide Scale (SOSS, short form): The short form SOSS will be used to assess stigmatising attitudes towards people who die by suicide. ${ }^{30}$ It consists of 16 one or two-word descriptors of a person who died by suicide, rated on a 5-point Likert scale ranging from (1) 'strongly disagree' to (5) 'strongly agree'. The SOSS has three subscales which assess stigma towards people who died by suicide, the normalisation or glorification of suicidal behaviour and the attribution of suicide to isolation. 
All subscales have shown good internal consistency in previous studies (Cronbach's alpha $>0.75$ ) ${ }^{31}$

Personal Suicide Stigma Questionnaire (PSSQ): The PSSQ differs from the SOSS as it assesses people's personal experiences of stigma related to suicidality in their own lives, rather than their beliefs about people who die by suicide. It consists of 16 items describing experiences of stigma, and participants are asked to rate how often those events have occurred, on a 5-point Likert scale from (1) 'never' to (5) 'very often'. It assesses three highly inter-related facets of suicide attempt stigma: rejection, minimisation and self-blame. The first two represent perceptions and experiences of stigma, while the third is similar to self-stigma. The items of the instrument are based on a qualitative study of personal stigma suffered by people who attempt suicide, and directly reflect lived experience. ${ }^{32}$ The questionnaire has demonstrated excellent internal consistency (Cronbach's alpha=0.95), is moderately positively correlated with the Self-Stigma of Mental Illness Scale $(\mathrm{r}=0.44, \mathrm{p}<0.001)$ and is strongly positively correlated with intensity of suicidal behaviour as assessed by the Suicide Behaviours Questionnaire $(\mathrm{r}=0.68, \mathrm{p}<0.001) .{ }^{33}$

Entrapment Scale: The Entrapment Scale consists of 16 items which reflect perceptions of feeling trapped and wishing to escape (eg, 'I can see no way out of my current situation'). Participants indicate the extent to which each item represents their own view of themselves on a 5-point scale from (0) 'not like me at all to (4) 'extremely like me'. The first six items relate to entrapment in internal thoughts and feelings (eg, 'I feel trapped inside myself'), while the last 10 relate to entrapment by external events or circumstances (eg, 'I am in a situation I feel trapped in'). Both subscales have demonstrated internal consistency (Cronbach's alpha $>0.85$ ), good test-retest reliability (intraclass correlation $($ ICC $)=0.90)^{34}$ and good convergent and criterion validity, being positively correlated with depression, hopelessness, submissive behaviour and suicidality in a number of separate studies. ${ }^{34-41}$

Distress Questionnaire-5 (DQ-5): The DQ-5 is a brief screener for psychological distress. It consists of five items each rated on a 5-point scale from (0) 'never' to (5) 'always', with total scores on the scale ranging from 5 to 25. Higher scores indicate greater psychological distress. The DQ-5 has shown good internal consistency (Cronbach's alpha $=0.86$ ) in a large community-based sample and demonstrates consistent performance across subgroups of age and gender. ${ }^{42}$

Alcohol Use Disorders Identification Test (AUDIT): The AUDIT is a widely used instrument that was developed by the WHO for identifying risky or harmful alcohol consumption. ${ }^{43}$ It consists of 10 questions which cover three domains: excessive alcohol intake, dependence and problems related to drinking. Each item is scored on a 5-point scale (0-4), with higher total scores indicating higher risk of alcohol abuse/addiction. The AUDIT has been widely validated ${ }^{445}$ and previous studies using this instrument showed that alcohol misuse is strongly associated with suicidality. ${ }^{46}$

Schuster Social Support Scale (SSSS): The SSSS is used to examine the quality of an individual's social relationships. ${ }^{47}$ Ten items from two subscales which measure support from friends and family will be used to measure the extent of positive and negative interactions (eg, "how often do your friends make you feel cared for?', 'how often do your family criticize you?'). Items are rated on a 4-point scale from (0) 'never' to (3) 'often'. All subscales have demonstrated acceptable internal reliability in separate studies (Cronbach's alpha 0.56-0.91). ${ }^{47} 48$

Composite Abuse Scale (Revised)-Short Form (CASR$\mathrm{SF})$ : The CASR-SF is a brief self-report measure of intimate partner violence. ${ }^{49}$ The 15 -item scale captures three abuse domains: physical, sexual and psychological, with questions used to assess severity and intensity of lifetime experiences (eg, 'Has this ever happened to you?'; 'IF YES, how often did it happen in the past 18 months?'). Endorsed items are rated on a 6-point scale from (0) 'not in the past 18 months' to (6) 'daily/almost daily'. The CASR-SF has shown good internal consistency (Cronbach's alpha $=0.94$ ) and has demonstrated initial reliability and validity for use in population-based studies. ${ }^{49}$

Two evidence-based screening items related to fear and control in current/previous relationships will be used to provide an indication of intimate partner violence (eg, 'In the past 18 months, how frequently have you experienced controlling behaviours in your current and/or past relationships?'). Response options are provided on a 5-point Likert scale from (0) 'never' to (5) 'frequently'. Only those participants who report experiencing fear or control in intimate relationships will receive the CASR-SF (eg, responses greater than 'rarely' will be flagged).

\section{Qualitative outcomes}

Semistructured interview guides have been developed for healthcare consumers, carers and health professionals, respectively. Questions are organised under predetermined concepts based on the literature ${ }^{12}$ (detailed below).

Healthcare consumers:

- Access to care.

- Assessment.

- Handover processes.

- Stigma, staff attitudes and staff knowledge.

- Patient involvement in treatment planning.

- Continuity of care.

- Discharge processes and follow-up.

- Aftercare. Carers:

- Access to care.

- Assessment.

- Handover processes.

- Inclusion of carer in treatment planning/assessment.

- Stigma, staff attitudes and staff knowledge.

- Continuity of care.

- Discharge processes and follow-up. 
- Support provided to carer.

Health professionals:

- Role and confidence when caring for people at risk of suicide.

- Standard of care for people at risk of suicide at the participant's organisation.

- Discharge from service and referral to aftercare.

- Barriers, problems and facilitating factors.

\section{Sample size}

Survey data

The population at risk has been defined as individuals aged 16 and over presenting to the ED following a suicidal crisis. Given fixed catchments and data collection periods, the attainable sample size is effectively fixed. HealthStats $\mathrm{NSW}^{50}$ for intentional self-harm hospitalisations (including ED-only presentations) for all ages in NSW recorded a total of 1168 presentations for trial sites and 1527 presentations for control sites for the 20172018 period. Given that cohort 1 includes ED presentations up to 1.5 years prior to assessment and cohort 2 includes ED presentations up to 12 months prior to assessment, there is a total eligibility period of 2.5 years for both cohorts. During this time frame based on data accessed from HealthStats ${ }^{50}$ as cited above it is estimated that approximately 2920 individuals in trial sites and 3817 individuals in control sites will be hospitalised for intentional self-harm.

Based on these figures, with a $20 \%-25 \%$ consent rate the attainable sample size would be $350-438$ people for trial sites and 458-527 people for control sites for cohort 1 and 233-292 people for trial sites and 305-381 people for cohort 2. These figures represent an estimated total sample size over the entire eligibility period of the study (2.5years) of 584-730 for trial sites and 763-954 for control sites. This estimate does not include hospital presentations for suicidal crisis without intentional selfharm, which could further increase the sample size.

As analysis for the current study will focus on betweencohort and between-site comparisons for the purpose of power analysis this study was treated as a cluster trial. This involves estimating a design effect arising from participants in each site potentially being more similar than between sites. ${ }^{51}$ Total sample sizes of approximately 700 people in the three trial sites and 900 people in the three control sites (as estimated above) result in a total sample of 1600 , with an average cluster size of 266 across the six sites. Conservatively assuming an ICC of 0.03 yields a design effect of 8.97. This 'deflates' the attainable sample size of 700 and 900 people by a factor of 9 resulting in an effective sample size of 78 and 100 people in trial sites and control sites, respectively. In this scenario, the study would have $80 \%$ power to detect a difference of $d=0.43$ between trial sites and control sites which represents a medium effect. However, it is recognised that given the complexity of the design these assumptions are difficult to assess. Participants within sites may well be generally more similar than between them on attributes such as demographic and socioeconomic characteristics. However, there is less evidence to suggest that this within-site similarity extends to factors such as personal attributes that may be related to the outcomes assessed within this trial. If this is the case, the observed ICC will be lower than allowed for in our calculations. Thus, achieved power may be greater than the estimate.

\section{Interview data}

For the current study Braun and Clarke's reflexive thematic analysis approach was adopted. In this approach meaning is generated through analysis, rather than extracted from the data. Determining the sample size in advance of data collection is difficult, as themes are not defined by a minimum number of occurrences or by a minimum number of participants mentioning the theme but rather as patterns of shared meaning across the data set. Following recommendations by Braun and Clarke for the current study a range was provided, rather than a fixed sample size. A benchmark for this range was the "Care After a Suicide Attempt' ${ }^{12}$ study for which 20 persons who had attempted suicide and 12 carers were interviewed about their healthcare experiences following a suicide attempt. Additionally, the breadth and focus of the research question and the expected perspectival diversity in the data were considered, as suggested by Braun and Clarke. ${ }^{52} 53$ The research questions for this study are organised in semistructured interview guides along predetermined domains and are thus quite focused. Based on the results of the "Care After a Suicide Attempt ${ }^{54}$ study mentioned above not much perspectival diversity was anticipated. Following these considerations, it was estimated that a range of 10-15 participants per group will be sufficient to generate complex patterns of meaning. The final sample size will be determined in situ, that is, during the data collection and analysis process.

\section{Data analysis}

\section{Survey data}

Data from the structured survey will be analysed quantitatively using appropriate statistical software. Exploratory analyses will be performed, including testing of assumptions of linearity, homoscedasticity, multicollinearity and normality of errors and, if necessary, transformation of data. Non-validated scales of the survey will be assessed for validity and reliability. $\chi^{2}$ tests for categorical outcome measures and t-tests for continuous outcome measures will be performed at baseline to examine the data for potential pre-existing differences between trial sites and control sites.

Mixed model repeated measures (MMRM) analysis of variance ${ }^{55}$ will be the principal means of comparing changes in primary and secondary outcomes between the LifeSpan and control sites. Such models allow the impact on outcomes of region/site factors, individual fixed and time-varying covariates to be taken into account. They also account for all available data under the missing-atrandom assumption. ${ }^{55}$ The primary test of the complex 
intervention will be the interaction between time and condition (intervention site vs control site), indicating a differential change in each outcome between intervention versus control sites over time. The secondary test of the intervention will be change in outcomes within intervention sites from the first wave to second wave of recruitment, to test the effects of maturation of implementation. It is hypothesised that patients in intervention sites will have greater improvement in outcomes over time compared with those in control sites, and that outcomes in intervention sites will improve from the first to the second wave. Linear MMRM will be used to assess changes in total YES scores (primary outcome). For variables that do not meet the assumptions of linear MMRM, analogous non-linear models, such as mixed effects logistic regression models, will be used to estimate changes (eg, proportion of participants reporting being referred to and taking up aftercare services, proportion of participants being followed up after discharge from the ED).

\section{Interview data}

Data from the qualitative interviews will be managed using NVivo. Data analysis will follow Clarke and Braun's reflexive thematic analysis approach, ${ }^{52}$ which is theoretically independent and can be used for a wide range of research questions, data collection and sampling methods. Themes are conceptualised as patterns of shared meaning across the data set, based on an underlying central concept or idea, that provides an answer to the research question (see research aim (iv): 'Understand the ED response to suicidal crisis from the perspectives of healthcare consumers, carers and health professionals'). Themes will be generated predominantly inductively from the data. However, some predetermined concepts will guide the first level of analysis. These are based on the literature, ${ }^{12}$ the study objectives and input from lived experience. The predetermined concepts are reflected in the interview questions. Data analysis will follow a rigorous process including several steps: (1) data familiarisation; (2) coding; (3) generating initial themes; (4) reviewing themes; (5) defining and naming themes; and (6) writing up. Coding will occur at two levels-semantic and latent with the objective of identifying themes across the whole data set.

\section{Status}

The project has commenced. Participants are currently being recruited to the study. Baseline data collection for the first cohort in survey participants will be finalised in September 2019. Baseline recruitment for the second cohort of survey participants will be finalised in September 2020. Baseline recruitment of interview participants will continue until the desired sample size has been reached for each group or until December 2019 (whichever occurs first).

\section{DISCUSSION}

The current study follows a group of healthcare consumers, carers and health professionals over time to explore the impact of the LifeSpan multilevel suicide prevention model on care provided following a suicidal crisis.

\section{Strengths}

The robust prospective cohort design will allow to determine the impact of LifeSpan on the ED response to suicidal crisis from a healthcare consumer perspective. Through inclusion of a qualitative component, the study will determine which elements of care are most highly valued by consumers, carers and providers and identify gaps and areas for improvement. The findings will be disseminated through existing partnerships built through an extensive collaborative effort as part of the LifeSpan suicide prevention model. Results from this study might be used to inform the provision of more effective and efficient models of care for people who engage in suicidal behaviours. This study follows global trends and national policy directions ${ }^{56}$ calling attention to the inclusion of persons with a lived experience in service design, planning and delivery.

\section{Limitations}

Random allocation of participants for this study in LifeSpan sites or control sites was not possible. However, cluster randomisation of LifeSpan sites has occurred within the broader LifeSpan trial to determine the order of crossover to the intervention. Additionally, details of crisis and aftercare policies and procedures in control sites are not known. Given the national emphasis on improving crisis care in EDs, ${ }^{56}$ some control sites may now have their own crisis and aftercare programmes in place. The Guidelines are based on evidence-based best-practice recommendations and it is likely that some aspects are incorporated into care at control sites. Further, only one LHD among the three control sites gave approval for face-to-face recruitment. This is not expected to significantly affect outcomes, as the majority of participants will be recruited via Facebook. Facebook has been reported to deliver similarly representative samples as traditional recruitment methods in the context of health, medical or psychosocial research. ${ }^{57}$

\section{Ethics and dissemination}

This research complies with national ethics standards and has been approved by the Hunter New England Human Research Ethics Committee (HREC/17/HNE/144) under the National Mutual Acceptance scheme. Further, the local research governance offices of Central Coast LHD, Murrumbidgee LHD, Illawarra Shoalhaven LHD and South Western Sydney LHD have given their approval for the study. Results will be disseminated via conferences and peer-reviewed journals.

\section{Confidentiality and Access to Data}

All personal data is managed in compliance with the Commonwealth Privacy Laws and the NSW Health Records 
and Information Privacy Act 2002 and will be de-identified before reporting. Data will be stored on secure passwordprotected servers, provided by the University of New South Wales, for a minimum of 15 years. The data is protected by an Active Directory Rights Management Service (AD RMS) and can only be accessed by principal and associate investigators listed on the ethics application for this study.

\section{Author affiliations}

${ }^{1}$ Black Dog Institute, Randwick, New South Wales, Australia

${ }^{2}$ Faculty of Medicine, University of New South Wales, Randwick, New South Wales, Australia

${ }^{3}$ Centre for Mental Health Research, Australian National University, Canberra, Australian Capital Territory, Australia

${ }^{4}$ School of Health, University of New England, Armidale, New South Wales, Australia ${ }^{5}$ Health Systems and Populations, Macquarie University, Sydney, New South Wales, Australia

Contributors FS, MT, ALC and PJB conceived the study. All authors contributed to the design of the study. FS is the chief investigator for this study. FS, MT, ALC, PJB and LS provided expertise in suicide prevention. AM and PJB provided expertise in statistics and quantitative data analysis. MM and LS provided expertise for design and analysis of the qualitative component of the study. HR is the research lead for this study and coordinated the development of the manuscript. HR and NC prepared the first manuscript. HR, NC, MT, ALC, PJB, LS, AM, MM, FS, HC and VMR contributed to the development of the manuscript by revising it critically for important intellectual content. All authors read and approved the final manuscript.

Funding This work is supported by a $\$ 14.76$ million grant from the Paul Ramsay Foundation for the period of 1 January 2016 until 31 December 2021. MT, ALC, PJB and FS are supported by National Health and Medical Research Council (NHMRC) Fellowships 1138710, 1122544, 1083311 and RG171846, respectively.

Disclaimer The design, management, analysis and reporting of the study are entirely independent of the Paul Ramsay Foundation.

\section{Competing interests None declared.}

Patient and public involvement Patients and/or the public were involved in the design, or conduct, or reporting, or dissemination plans of this research. Refer to the Methods section for further details.

Patient consent for publication Not required.

Provenance and peer review Not commissioned; externally peer reviewed.

Open access This is an open access article distributed in accordance with the Creative Commons Attribution Non Commercial (CC BY-NC 4.0) license, which permits others to distribute, remix, adapt, build upon this work non-commercially, and license their derivative works on different terms, provided the original work is properly cited, appropriate credit is given, any changes made indicated, and the use is non-commercial. See: http://creativecommons.org/licenses/by-nc/4.0/.

\section{ORCID iD}

Hannah Rosebrock http://orcid.org/0000-0003-0650-0711

\section{REFERENCES}

1 Australian Bureau of Statistics. Causes of death, Australia, 2015, cat no 3303.0, 2016

2 Hoven CW, Wasserman D, Wasserman C, et al. Awareness in nine countries: a public health approach to suicide prevention. Leg Med 2009;11 Suppl 1:S13-17.

3 De Leo D, Cerin E, Spathonis K, et al. Lifetime risk of suicide ideation and attempts in an Australian community: prevalence, suicidal process, and help-seeking behaviour. J Affect Disord 2005;86:215-24.

4 Reith DM, Whyte I, Carter G, et al. Risk factors for suicide and other deaths following Hospital treated self-poisoning in Australia. Aust $N$ Z J Psychiatry 2004;38:520-5.

5 World Health Organisation. Preventing suicide: a global imperative, 2014.

6 Owens D, Horrocks J, House A. Fatal and non-fatal repitition of selfharm: systematic review. Br J Psychiatry 2002;181.
7 Bergen $\mathrm{H}$, Hawton K, Waters K, et al. Premature death after selfharm: a multicentre cohort study. Lancet 2012;380:1568-74.

8 Christiansen E, Jensen BF. Risk of repetition of suicide attempt, suicide or all deaths after an episode of attempted suicide: a register-based survival analysis. Aust $N Z J$ Psychiatry 2007;41:257-65.

9 Hunt IM, Kapur N, Webb R, et al. Suicide in recently discharged psychiatric patients: a case-control study. Psychol Med 2009;39:443-9.

10 Chung D, Hadzi-Pavlovic D, Wang M, et al. Meta-Analysis of suicide rates in the first week and the first month after psychiatric hospitalisation. BMJ Open 2019;9:e023883.

11 Krysinska K, Batterham PJ, Tye M, et al. Best strategies for reducing the suicide rate in Australia. Aust N Z J Psychiatry 2016;50:115-8.

12 NHMRC. NHMRC centre of research excellence in suicide prevention (CR.E.S.P). care after a suicide attempt, 2015.

13 SANE Australia UoNE. Lessons for life: the experiences of people who attempt suicide: a qualitative research report. Australia, 015.

14 Hegerl U, Althaus D, Schmidtke A, et al. The alliance against depression: 2-year evaluation of a community-based intervention to reduce suicidality. Psychol Med 2006;36:1225-33.

15 Hegerl U, Wittmann M, Arensman E, et al. The 'European Alliance Against Depression (EAAD)': a multifaceted, community-based action programme against depression and suicidality. World J Biol Psychiatry 2008;9:51-8.

16 Shand F, Torok M, Cockayne N, et al. Protocol for a stepped-wedge, cluster randomized controlled trial of the lifespan suicide prevention trial in four communities in New South Wales, Australia. Trials 2020;21:332.

17 Shand F, Vogl L, Robinson J. Improving patient care after a suicide attempt. Australas Psychiatry 2018;26:145-8.

18 Brown GK, Green KL. A review of evidence-based follow-up care for suicide prevention: where do we go from here? Am J Prev Med 2014;47:S209-15.

19 Hill NTM, Shand F, Torok M, et al. Development of best practice guidelines for suicide-related crisis response and aftercare in the emergency department or other acute settings: a Delphi expert consensus study. BMC Psychiatry 2019;19:6.

20 Hill N, Halliday L, Reavley NJ. Guidelines for integrated suiciderelated crisis and follow-up care in emergency departments and other acute settings. in. Australia: Black Dog Institute, 2017.

21 Shekelle PG, Maglione MA, Luoto J, et al. Global Health Evidence Evaluation Framework [Internet]. Rockville (MD): Agency for Healthcare Research and Quality (US); 2013 Jan. Table B.9, NHMRC Evidence Hierarchy: designations of 'levels of evidence' according to type of research question (including explanatory notes), 2013. Available: https://www.ncbi.nlm.nih.gov/books/NBK121300/table/ appb.t21/

22 Song JW, Chung KC. Observational studies: cohort and case-control studies. Plast Reconstr Surg 2010;126:2234-42.

23 Curry LA, Nembhard IM, Bradley EH. Qualitative and mixed methods provide unique contributions to outcomes research. Circulation 2009;119:1442-52.

24 Pope C, van Royen P, Baker R. Qualitative methods in research on healthcare quality. Qual Saf Health Care 2002;11:148-52.

25 Tye M, Shand F, Krysinska K, et al. A systems approach to suicide prevention: Implementation Plan - Protocol version 2. Sydney: Black Dog Institute for the NSW Mental Health Commission, 2016.

26 Your Experience of Service. Australia's national mental health consumer experience of care survey. in: health AGDo, 2015

27 Mundt JC, Greist JH, Jefferson JW, et al. Prediction of suicidal behavior in clinical research by lifetime suicidal ideation and behavior ascertained by the electronic Columbia-Suicide severity rating scale. $J$ Clin Psychiatry 2013;74:887-93.

28 Greist JH, Mundt JC, Gwaltney CJ, et al. Predictive value of baseline electronic Columbia-Suicide severity rating scale (eC-SSRS) assessments for identifying risk of prospective reports of suicidal behavior during research participation. Innov Clin Neurosci

29 Rickwood D, Deane FP, Wilson CJ, et al. Young people's helpseeking for mental health problems. Australian e-Journal for the Advancement of Mental Health 2005;4:218-51.

30 Batterham PJ, Calear AL, Christensen H. Correlates of suicide stigma and suicide literacy in the community. Suicide Life Threat Behav 2013;43:406-17.

31 Calear AL, Batterham PJ, Christensen H. Predictors of help-seeking for suicidal ideation in the community: risks and opportunities for public suicide prevention campaigns. Psychiatry Res 2014;219:525-30.

32 Rimkeviciene J, Hawgood J, O'Gorman J, et al. Personal stigma in suicide Attempters. Death Stud 2015;39:592-9. 
33 Rimkeviciene J, O'Gorman J, Hawgood J, et al. Development and validity of the personal suicide stigma questionnaire (PSSQ). Crisis 2019;40:317-25.

34 Taylor PJ, Gooding P, Wood AM, et al. The role of defeat and entrapment in depression, anxiety, and suicide. Psychol Bull 2011;137:391-420.

35 Gilbert P, Allan S. The role of defeat and entrapment (arrested flight) in depression: an exploration of an evolutionary view. Psychol Med 1998;28:585-98.

36 Allan S, Gilbert P. Anger and anger expression in relation to perceptions of social RANK, entrapment and depressive symptoms. Pers Individ Dif 2002;32:551-65.

37 Trachsel M, Krieger T, Gilbert P, et al. Testing a German adaption of the entrapment scale and assessing the relation to depression. Depress Res Treat 2010;2010:1-10.

38 Taylor PJ, Wood AM, Gooding P, et al. Appraisals and suicidality: the mediating role of defeat and entrapment. Arch Suicide Res 2010;14:236-47.

39 Griffiths AW, Wood AM, Maltby J, et al. The prospective role of defeat and entrapment in depression and anxiety: a 12-month longitudinal study. Psychiatry Res 2014;216:52-9.

40 O'Connor RC, Smyth R, Ferguson E, et al. Psychological processes and repeat suicidal behavior: a four-year prospective study. $J$ Consult Clin Psychol 2013;81:1137-43.

41 Ng RMK, Di Simplicio M, McManus F, et al. 'Flash-forwards' and suicidal ideation: a prospective investigation of mental imagery, entrapment and defeat in a cohort from the Hong Kong mental morbidity survey. Psychiatry Res 2016;246:453-60.

42 Batterham PJ, Sunderland M, Carragher N, et al. The distress Questionnaire-5: population screener for psychological distress was more accurate than the K6/K10. J Clin Epidemiol 2016;71:35-42.

43 Saunders JB, Aasland OG, Babor TF, et al. Development of the Alcohol Use Disorders Identification Test (AUDIT): WHO Collaborative Project on Early Detection of Persons with Harmful Alcohol Consumption--II. Addiction 1993;88:791-804.

44 Reinert DF, Allen JP. The alcohol use disorders identification test (audit): a review of recent research. Alcohol Clin Exp Res 2002;26:272-9.

45 de Meneses-Gaya C, Zuardi AW, Loureiro SR, et al. Alcohol use disorders identification test (audit): an updated systematic review of psychometric properties. Psychol Neurosci 2009;2:83-97.
46 McCloud A, Barnaby B, Omu N, et al. Relationship between alcohol use disorders and suicidality in a psychiatric population: in-patient prevalence study. Br J Psychiatry 2004;184:439-45.

47 Schuster TL, Kessler RC, Aseltine RH. Supportive interactions, negative interactions, and depressed mood. Am J Community Psychol 1990;18:423-38.

48 DWY L, Fairweather-Schmidt AK, Burns RA, et al. The utility of nonspecific measures of resilience across the lifespan: an investigation of structural invariance across gender and age cohorts. Australian Journal of Psychology 2016;68:3-10.

49 Ford-Gilboe M, Wathen CN, Varcoe C, et al. Development of a brief measure of intimate partner violence experiences: the Composite Abuse Scale (Revised)-Short Form (CASR-SF). BMJ Open 2016;6:e012824.

50 HealthStats NSW. Intentional self-harm: hospitalisations by local health district and year, 2017. Available: http://www.healthstats.nsw. gov.au/Indicator/men_suihos/men_suihos_Ihn [Accessed 09 Apr 2019].

51 Rutterford C, Copas A, Eldridge S. Methods for sample size determination in cluster randomized trials. Int J Epidemiol 2015;44:1051-67.

52 Braun V, Clarke V, Hayfield N, et al. Thematic analysis. In: Liamputtong $\mathrm{P}$, ed. Handbook of research methods in health socia sciences. Singapore: Springer Singapore, 2019: 843-60.

53 Braun V, Clarke V. To saturate or not to saturate? Questioning data saturation as a useful concept for thematic analysis and sample-size rationales. Qual Res Sport Exerc Health 2019;2:1-16.

54 Shand FL, Batterham PJ, Chan JKY, et al. Experience of health care services after a suicide attempt: results from an online survey. Suicide Life Threat Behav 2018;48:779-87.

55 Verbeke G, Molenberghs G. Linear mixed models for longitudinal data. Springer, 2000

56 Australian Department of Health. The Fith national mental health and suicide prevention plan. Canberra, Australia: Commonwealth of Australia Department of Health, 2017

57 Thornton L, Batterham PJ, Fassnacht DB, et al. Recruiting for health, medical or psychosocial research using Facebook: systematic review. Internet Interv 2016;4:72-81. 